Article

\title{
Abscisic Acid (ABA) Regulation of Arabidopsis SR Protein Gene Expression
}

\author{
Tiago M. D. Cruz, Raquel F. Carvalho, Dale N. Richardson and Paula Duque * \\ Instituto Gulbenkian de Ciência, Rua da Quinta Grande 6, Oeiras 2780-156, Portugal; \\ E-Mails: tcruz@igc.gulbenkian.pt (T.M.D.C.); rcarvalho@igc.gulbenkian.pt (R.F.C.); \\ drichardson@igc.gulbenkian.pt (D.N.R.)
}

* Author to whom correspondence should be addressed; E-Mail: duquep@igc.gulbenkian.pt; Tel.: +351-21-446-4630; Fax: +351-21-440-7970.

External Editor: Akila Mayeda

Received: 2 August 2014; in revised form: 13 September 2014 / Accepted: 23 September 2014 /

Published: 29 September 2014

\begin{abstract}
Serine/arginine-rich (SR) proteins are major modulators of alternative splicing, a key generator of proteomic diversity and flexible means of regulating gene expression likely to be crucial in plant environmental responses. Indeed, mounting evidence implicates splicing factors in signal transduction of the abscisic acid (ABA) phytohormone, which plays pivotal roles in the response to various abiotic stresses. Using real-time RT-qPCR, we analyzed total steady-state transcript levels of the $18 \mathrm{SR}$ and two SR-like genes from Arabidopsis thaliana in seedlings treated with ABA and in genetic backgrounds with altered expression of the ABA-biosynthesis $A B A 2$ and the ABA-signaling $A B I 1$ and $A B I 4$ genes. We also searched for ABA-responsive cis elements in the upstream regions of the 20 genes. We found that members of the plant-specific SC35-Like (SCL) Arabidopsis SR protein subfamily are distinctively responsive to exogenous ABA, while the expression of seven SR and SR-related genes is affected by alterations in key components of the ABA pathway. Finally, despite pervasiveness of established ABA-responsive promoter elements in Arabidopsis SR and SR-like genes, their expression is likely governed by additional, yet unidentified cis-acting elements. Overall, this study pinpoints $S R 34, S R 34 b, S C L 30 a, S C L 28$, $S C L 33, R S 40, S R 45$ and $S R 45 a$ as promising candidates for involvement in ABA-mediated stress responses.
\end{abstract}


Keywords: abscisic acid (ABA); ABA-responsive cis elements; alternative splicing (AS); Arabidopsis thaliana; gene expression; SR proteins

\section{Introduction}

Precursor messenger RNA (pre-mRNA) splicing is an essential step in gene expression mediated by the spliceosome, a large protein complex in the cell nucleus that interacts with specific intronic sequences in the pre-mRNA called splice sites for the proper removal of introns and correct joining of exons. Alternative splicing (AS) occurs when splice sites are differentially recognized, allowing for the production of multiple transcripts from a single gene that can potentially result in different protein isoforms. In addition to largely expanding the coding capacity of genomes, AS represents an important means of regulating gene expression, for instance by introducing premature termination codons (PTCs) that then target these transcripts for degradation by a process known as nonsense-mediated mRNA decay (NMD) [1].

Serine/arginine-rich (SR) proteins constitute a highly conserved family of RNA-binding proteins (RBPs) with key roles in constitutive, and particularly AS, by affecting splice site selection in a concentration- and phosphorylation-dependent manner [2]. Their distinctive protein domain organization consists of one or two $N$-terminal RNA recognition motifs (RRMs) and an arginine/serine-rich domain (RS) located at the $C$-terminus [3]. The RRM binds the pre-mRNA conferring target specificity [4] whereas the RS domain, which can be highly phosphorylated at multiple serine residues [5], is involved in protein-protein interactions allowing the recruitment of core components of the splicing machinery $[4,6]$. SR proteins act by binding auxiliary cis-acting regulatory elements, such as exonic or intronic splicing enhancers or silencers (ESE/ESS; ISE/ISS), thereby influencing splice site choice and bridging spliceosomal components at the 5' and 3' splice sites [7]. Additional roles have been uncovered in animal systems for these splicing factors, including in mRNA nuclear export, NMD and translation, or in genome stability, transcriptional elongation and microRNA processing [7-10].

Despite considerable progress in recent years, the functions of plant SR proteins remain less understood than those of their metazoan counterparts. The Arabidopsis thaliana genome codes for 18 SR proteins that can be grouped into six subfamilies. The SR, RSZ and SC subfamilies include direct orthologs of the mammalian SR splicing factors SRSF1, SRSF7 and SRSF2, respectively, while the SCL, RS2Z and RS subfamilies are plant-specific. Additionally, the Arabidopsis genome encodes two SR-like proteins, SR45 and SR45a, which despite sharing properties with other SRs display an atypical domain organization (two RS domains flanking an RRM) that does not meet the accepted SR protein nomenclature criteria [11]. A few Arabidopsis SR and SR-like proteins have been shown to function as bona fide splicing factors [12,13] and to play in vivo roles in plant development and/or responses to environmental cues [13-17]. Importantly, several have also been found to target AS of multiple pre-mRNAs, including those of other members of the SR gene family $[13-15,18,19]$.

AS is pervasive in higher eukaryotes and has long been known to determine key biological processes in animal systems [20,21]. In humans, about 95\% of the multi-exon genes undergo AS [22,23], of which misregulation is associated with numerous severe diseases [24,25]. In higher plants, the 
first transcriptome-wide analyses indicate that half or more of the intron-containing genes are alternatively-spliced [26,27], with the current estimate in Arabidopsis plants grown under normal conditions being above $60 \%$ [28]. However, the biological relevance of AS in plants is just beginning to unfold, namely in developmental processes, as well as in the response to pathogens and, particularly, abiotic stress [29-31]. In fact, stress-associated genes are particularly prone to AS [32-34], which is markedly affected by abiotic stresses [32,35-39], and the expression/splicing pattern of several SR or SR-like proteins is stress-regulated [38,40-42], pointing to a crucial role for AS in plant responses to environmental stress.

Abscisic acid (ABA) is a major plant hormone playing a key role as an abiotic stress response modulator. Indeed, ABA is crucial in the coordination of several signal transduction pathways involved in the response to environmental stresses such as drought, high salinity, cold and heat, all of which are known to increase endogenous ABA levels [43,44]. Currently, the core ABA signaling network is comprised of 13 pyrabactin resistance 1-like (PYL) receptors, nine A-type protein phosphatase 2Cs (PP2Cs), three sucrose nonfermenting-1-related protein kinase 2s (SNRK2s) and five basic leucine zipper transcriptional activators known as ABA-responsive element binding factors (ABFs) $[45,46]$. However, recent systems biology approaches have uncovered an ABA signaling network of over 500 interactions among 138 different proteins [47]. Transcriptional activation of downstream genes responsive to $\mathrm{ABA}$ is governed by phosphorylation of the $\mathrm{ABFs}$, which bind to cis elements in target gene promoters known as ABREs (ABA-responsive elements) [45] to activate gene expression. While the core ABFs play an important role in ABRE-dependent gene expression, many genes induced by ABA lack ABRE elements and the transcription factors that activate these genes have yet to be clearly delineated [47].

Interestingly, emerging evidence is linking plant RBPs, and splicing factors in particular, to ABA-mediated stress responses. The conserved splicing factor Suppressor of abi3-5 (SUA) was found to influence seed ABA sensitivity in Arabidopsis by regulating AS of the ABI3 signaling gene [48]. Similarly, a recent study showed that AtSF1, the Arabidopsis homolog of the mammalian SF1 splicing factor, is active in AS and regulates sensitivity to ABA [49]. ABA-related phenotypes have also been described for mutants in plant heterogeneous nuclear ribonucleoproteins (hnRNPs) [50-52], another class of RBPs active in AS. Regarding the SR protein family, Palusa et al. [40] showed that exogenous ABA application slightly alters the AS pattern of three Arabidopsis SR genes, and Xiong and co-workers [17] have identified ABA and salt stress hypersensitive phenotypes upon deletion of two members of the plant-specific RS subfamily, RS40 and RS41. A knockout mutant for a member of the SCL subfamily, SCL30a, is also hypersensitive to ABA during seed germination (our unpublished results). Finally, the SR-like SR45 protein negatively regulates glucose signaling during early seedling development in Arabidopsis by downregulating the ABA pathway [16]. These findings suggest that posttranscriptional networks may act as central coordinators of plant abiotic stress responses by targeting key components of the ABA signal transduction machinery.

To investigate the extent of SR gene expression regulation by the stress phytohormone ABA, we have analyzed total transcript levels of the 20 Arabidopsis thaliana SR and SR-like genes by real-time RT-qPCR in plants treated exogenously with ABA and in different ABA-related genetic backgrounds. Furthermore, to gain insight into which SR and SR-like genes may respond to the core 
ABFs, we also examined upstream sequences for each of the SR and SR-like genes for occurrences of putative ABRE cis elements.

\section{Results and Discussion}

\subsection{Effect of Exogenous Abscisic Acid (ABA) on the Expression of Arabidopsis Serine/Arginine-Rich (SR) and SR-Like Genes}

To study the effect of the stress phytohormone ABA on total steady-state transcript levels of SR and SR-like genes, we treated 15 day-old Arabidopsis seedlings from the Columbia (Col-0) ecotype with $50 \mu \mathrm{M} \mathrm{ABA}$ for $5 \mathrm{~h}$ and performed real-time RT-qPCR. To ensure that the treatment was effective, RT-qPCR analyses were first performed with primers specific to the ABA-responsive gene RD29A [53]. Indeed, as seen in Figure 1, a six-fold increase in $R D 29 A$ expression levels was observed, indicating that the ABA treatment worked.

Figure 1. Effect of the abscisic acid (ABA) treatment on $R D 29 A$ gene expression. Real-time RT-qPCR analysis of total transcript levels of the ABA-responsive gene $R D 29 A$ in control and ABA-treated (50 $\mu \mathrm{M}$ for $5 \mathrm{~h})$ Arabidopsis seedlings. Results are from two independent experiments and values represent means $\pm \mathrm{SE}(n=4)$. Asterisks indicate a significant difference $(* * * p<0.001$; Student's $t$-test) from control plants.

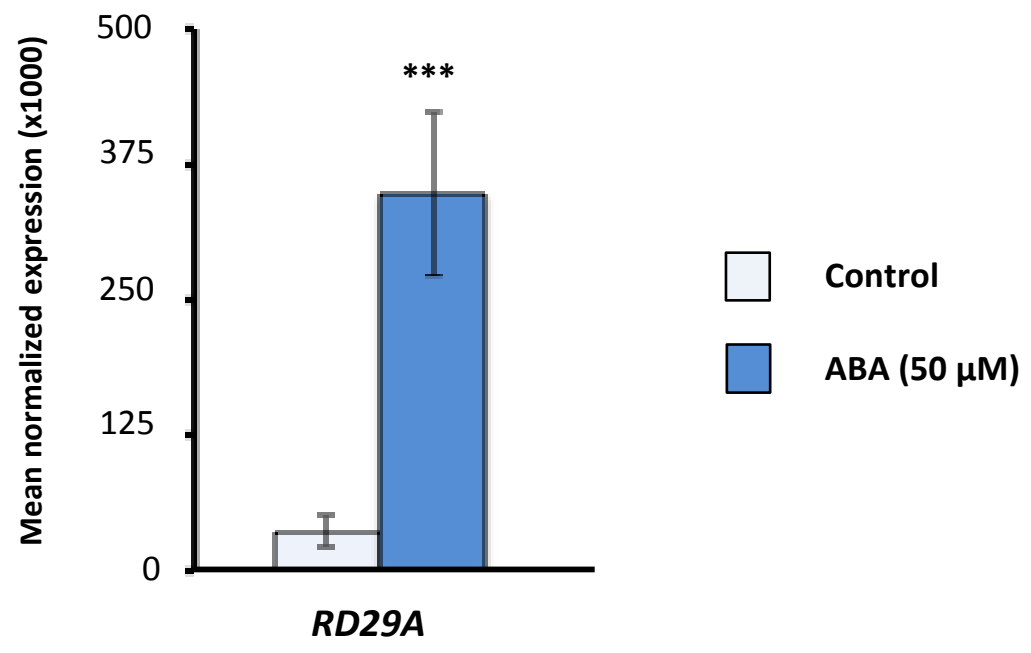

As shown in Figure 2A,B, among the 18 Arabidopsis SR genes, only three members of the plant-specific SCL subfamily (SCL30a, SCL28 and SCL33) had their expression patterns significantly altered $(p<0.05)$. While SCL28 expression was induced by ABA, SCL33 and SCL30a-Two gene paralogs located on duplicated regions of the genome [54,55]-Were repressed (Figure 2B). The expression profile of these three SR genes suggests they could very likely be involved in ABA responses. In agreement with our results, the SCL33 gene has been previously described as being downregulated by ABA but, contrary to what we report, SCL28 and SCL3Oa were found to be unaffected by $\mathrm{ABA}$ treatment [40]. Previous studies using whole genome tiling arrays have shown that $\mathrm{ABA}$ treatments for different periods of time activate different genes [56,57], and this could explain why SCL3Oa and SCL28 behaved differently in our study, as not only the ABA concentration but also the time points used were different from those reported by Palusa et al. [40]. On the other hand, 
the induction seen in SCL28 transcript levels may also be the result of a more accurate quantification through real-time RT-qPCR, as the expression levels for this gene are very low and can hardly be detected by semi-quantitative RT-PCR. Given that none of the members from the other SR gene subfamilies exhibited misregulation upon ABA treatment, the plant-specific SCL subfamily may be distinctly responsive to this stress phytohormone.

Figure 2. Effect of ABA treatment on the expression of Arabidopsis serine/arginine-rich (SR) and SR-like genes. Real-time RT-qPCR analysis of total transcript levels of SR gene members of the mammalian ortholog (A) and plant-specific (B) subfamilies or of SR-like genes $(\mathbf{C})$ in control and ABA-treated $(50 \mu \mathrm{M}$ for $5 \mathrm{~h}$ ) wild-type (Col-0) Arabidopsis seedlings. Results are from two independent experiments and values represent means $\pm \mathrm{SE}$ $(n=4)$. Asterisks indicate significant differences $(* * p<0.01, * * * p<0.001$; Student's $t$-test) from the corresponding wild type.

(A)

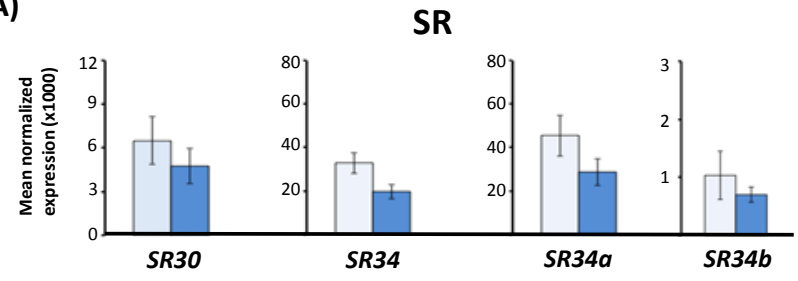

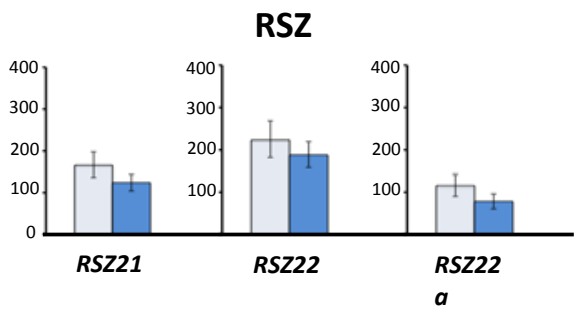

RS2Z

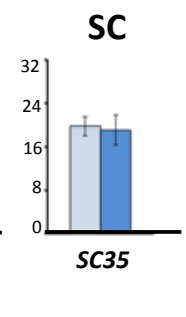

RS

(B)
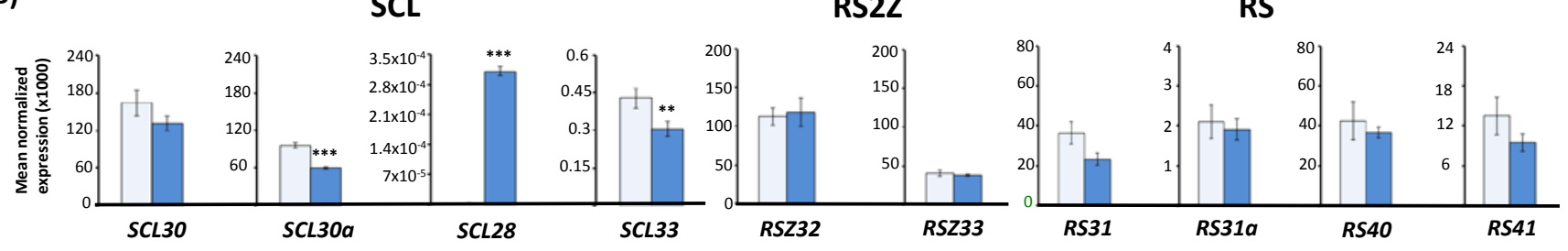

(C)

\section{SR-like}

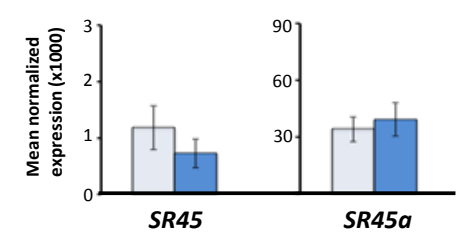

Control

ABA $(50 \mu M)$

Regarding the SR-like genes, neither $S R 45$ nor $S R 45$ a gene expression was affected by the exogenous application of ABA (Figure 2C). Indeed, the expression of $S R 45$ has been reported to show significant changes under sucrose and temperature stress [58], but not in response to ABA or other phytohormones [40,58]. Expression of $S R 45 a$ is markedly induced by high light stress [59], while DNA microarray studies [60-63] and RNA-seq analysis of abiotic stress samples [64] have shown that SR45a mRNA abundance is also altered upon heat and water deprivation stresses. Nevertheless, SR45a expression in response to ABA was not investigated until this study.

\subsection{Expression of Arabidopsis SR and SR-Like Genes in the aba2-1 Mutant Background}

After studying the effect of exogenously applied ABA on the expression levels of SR and SR-like genes, we next asked whether a reduced endogenous plant ABA content would affect these genes. 
To address this question, we compared total SR and SR-like transcript levels in wild-type (Col-0) seedlings and those from the ABA-deficient mutant aba2-1 [65] (Figure 3). The ABA2 gene encodes a short-chain alcohol dehydrogenase/reductase (SDR) protein that catalyzes the conversion of xanthoxin to abscisic aldehyde in the ABA biosynthesis pathway, and its loss of function leads to substantially reduced ABA levels [65-68].

Figure 3. Expression of Arabidopsis SR and SR-like genes in the ABA-deficient aba2-1 mutant background. Real-time RT-qPCR analysis of total transcript levels of SR gene members of the mammalian ortholog (A) and plant-specific (B) subfamilies or of SR-like genes $(\mathbf{C})$ in wild-type (Col-0) and aba2-1 mutant seedlings. Results are from two independent experiments and values represent means \pm SE $(n=4)$. Asterisks indicate significant differences $(* p<0.05, * * p<0.01$; Student's $t$-test) from the corresponding wild type.

(A)

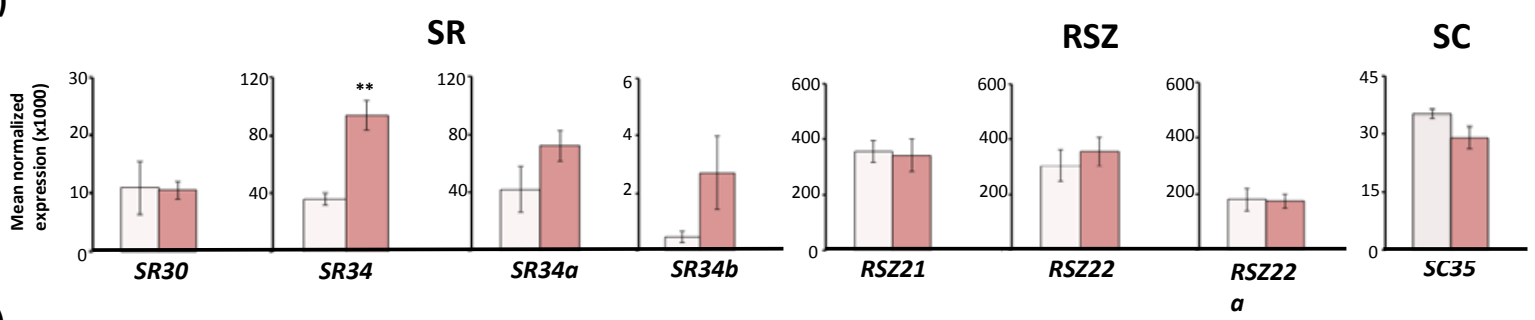

(B)

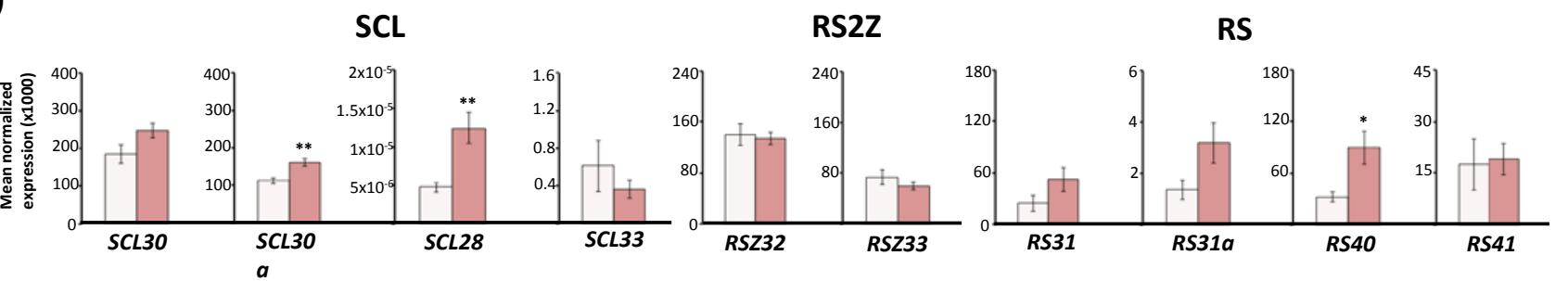

(C)

\section{SR-like}

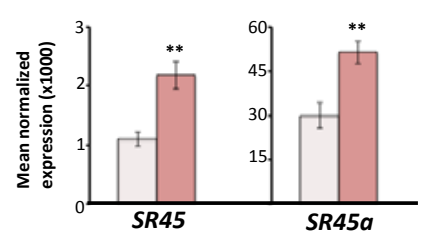

Col-0

$a b a 2-1$

From the mammalian ortholog Arabidopsis SR subfamilies, only expression of the SR34 gene was altered in aba2-1, displaying higher steady state transcript levels in this mutant background (Figure 3A). Although still not functionally characterized, the $S R 34$ gene was previously reported to be repressed by exogenous ABA [40]. That SR34 expression was upregulated in the aba2-1 mutant lends further credence to the notion that this SR gene is modulated by the phytohormone.

Three SR plant-specific genes were affected in the aba2-1 mutant background: SCL30a and SCL28, members of the SCL subfamily, and RS40 from the RS subfamily (Figure 3B). All three genes exhibited higher transcript levels in the mutant when compared to the wild type. The upregulation of the SCL30a gene in aba2-1 seedlings is concordant with its repression by ABA treatment (see Figure 2B) and with previously produced data in our lab supporting SCL30a as a negative regulator of ABA-dependent salt and drought stresses during seed germination (unpublished results). According to the Arabidopsis electronic Fluorescent Pictograph (eFP) browser [69], SCL28 expression is low at almost all 
phases of the Arabidopsis life cycle. Intriguingly, this SR gene was induced both by exogenous ABA (see Figure 2B) and in the aba2-1 mutant background. This may indicate that SCL28 expression is under tight ABA control, with slight changes in endogenous basal hormone levels being sufficient to disrupt this regulation. The third plant-specific SR gene affected in the aba2-1 mutant, RS40, was very recently implicated in the ABA pathway. In fact, knockout mutants for $R S 40$ or its duplicated gene pair $R S 41$ were found to display ABA hypersensitivity during seed germination and early seedling development [17]. The same study reports that expression of both these SR genes is slightly ABA induced, but their transcript levels were not significantly changed by the phytohormone under our experimental conditions (see Figure 2B).

Finally, expression of the two SR-related genes, $S R 45$ and $S R 45 a$, was noticeably induced in the aba2-1 mutant background (Figure 3C). A loss of function mutant for SR 45, sr45-1, is hypersensitive to glucose and, similarly to the $r s 40$ and $r s 41$ mutants, also displays an oversensitive response to the exogenous application of ABA [16]. Moreover, the $s r 45-1$ mutation causes enhanced glucose-specific ABA accumulation, as well as ABA biosynthesis and signaling gene expression [16]. The observation that $S R 45$ is upregulated in plants impaired in ABA synthesis suggests that ABA represses the gene's expression in vivo. This could be important for amplification of ABA signal transduction under stress conditions, where enhanced production of ABA would reduce the levels of its negative regulator SR45, thereby releasing repression of ABA-mediated inhibition of early seedling development. Future detailed characterization of the Arabidopsis SR45a gene, which was also markedly upregulated in the aba2-1 background, should reveal whether it fulfills similar ABA-related functions.

\subsection{Expression of Arabidopsis SR and SR-Like Genes in the abi1-1, abi4-101 and ABI4OX Backgrounds}

In addition to exogenous $\mathrm{ABA}$ application and reduced $\mathrm{ABA}$ biosynthesis, we also investigated if alterations in the levels of ABA signaling components could affect SR and SR-like gene expression. To evaluate this hypothesis, we checked the total transcript levels of Arabidopsis SR and SR-like genes in the ABA-insensitive abil-1 [70] and abi4-101 [71] mutants, as well as in an ABI4-overexpressing ( $A B I 4 \mathrm{OX}$ ) transgenic line [72] (Figures 4 and 5). The $A B I 1$ gene encodes protein phosphatase 2C (PP2C), a membrane protein that plays a key role in ABA sensing and signaling [73,74]. $\mathrm{ABI} 4$ is a versatile ethylene responsive factor/APetala2 (ERF/AP2) transcription factor essential for ABA-dependent transcriptional changes that acts both as an activator and a repressor of gene expression [75]. Both genes mediate ABA signal transduction but while $A B I 4$ function has been primarily described in seed tissues and during early seedling development, $A B I 1$ is also known to play crucial roles at the vegetative phase namely in the regulation of stomatal closure.

As depicted in Figure 4A,B, of all the Arabidopsis SR genes only SR34b exhibited altered expression in the abil-1 mutant $(p<0.05)$. In fact, although several RBP-encoding genes were found to be misregulated in a genomewide expression profiling of Arabidopsis wild-type (Ler ecotype) versus abil-1 mutant seedlings, no changes in SR or SR-like genes were noticed [76]. Our observed repression of $S R 34 b$ in the abil-1 background suggests that expression of this gene is under $A B I 1$-signaling control, pointing to a role in ABA-mediated responses. Indeed, and although here total $S R 34 b$ expression was unaffected by ABA treatment (see Figure 2), Palusa et al. [40] reported that AS of the $S R 34 b$ pre-mRNA is altered by exogenous $\mathrm{ABA}$ and environmental stresses. Interestingly, the Arabidopsis splicing factor 
SUA was found to control ABA-mediated seed responses by specifically regulating AS of an another ABI signaling gene, $A B I 3$ [48]. Whether $S R 34 b$ plays any role in splicing of the $A B I 1$ pre-mRNA remains to be elucidated-What our results do indicate is that ABI1 is involved in promoting expression of the SR34b splicing factor.

As for the two Arabidopsis SR-like genes, while SR45 expression remained unchanged, SR45a was downregulated nearly two fold in the abil-1 mutant background (Figure 4C). This again strongly suggests that $S R 45 a$ expression is targeted by ABI1-mediated signaling, holding much promise for the future functional characterization of this SR-related gene in ABA responses.

Figure 4. Expression of Arabidopsis SR and SR-like genes in the ABA-signaling abil-1 mutant background. Real-time RT-qPCR analysis of total transcript levels of SR gene members of the mammalian ortholog (A) and plant-specific (B) subfamilies or of SR-like genes (C) in wild-type (Ler) and abil-1 mutant seedlings. Results are from two independent experiments and values represent means \pm SE $(n=4)$. Asterisks indicate significant differences $(* p<0.05$; Student's $t$-test) from the corresponding wild type.

(A)

SR

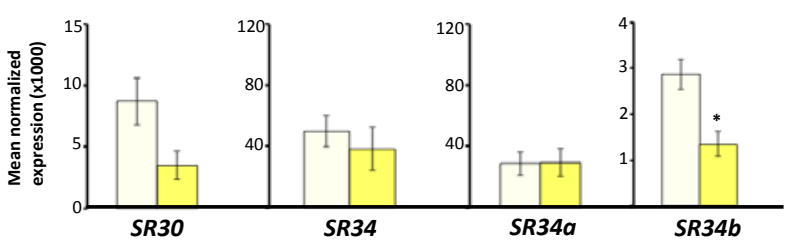

(B)

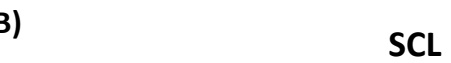

$\mathrm{SCL}$

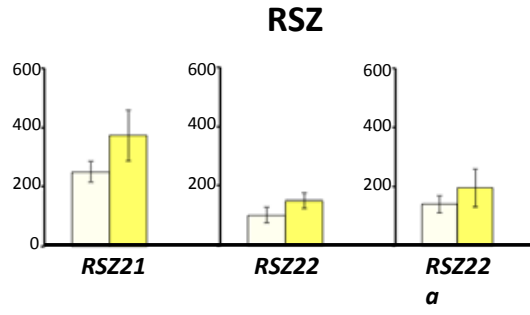

SC
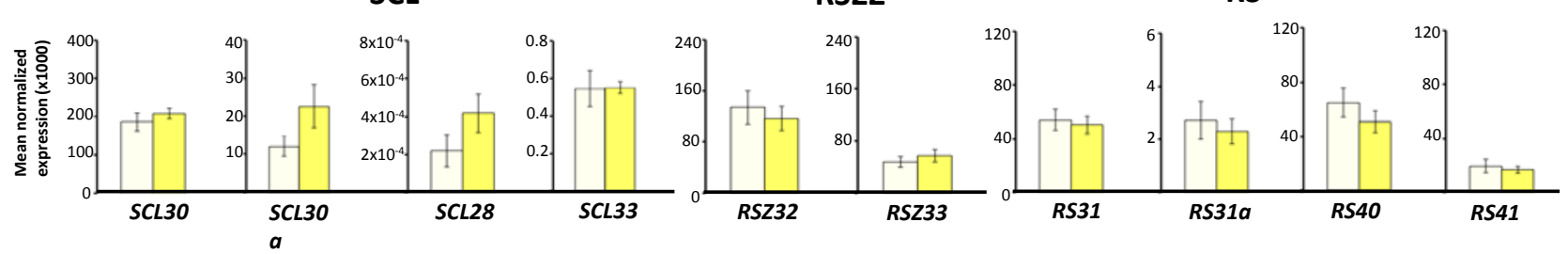

(C)

SR-like

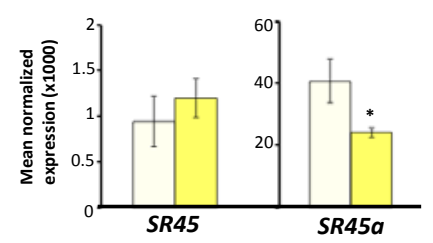

Finally, we analyzed the expression patterns of Arabidopsis SR and SR-like genes in the abi4-101 and $A B I 4 \mathrm{OX}$ backgrounds (Figure 5). We found no changes in the total transcript levels of any of the SR or SR-like genes in seedlings of either $A B I 4$ genotype $(p<0.05)$, except for the $S R 34 b$ gene, belonging to the SR subfamily of orthologs of the mammalian splicing factor SRSF1, and for the plant-specific $R S 40$ gene (Figure 5A,B). Surprisingly, both loss and gain of $A B I 4$ function led to induction of $S R 34 b$ and $R S 40$ expression. 
Figure 5. Expression of Arabidopsis SR and SR-like genes in the ABA-signaling abi4-101 mutant and $A B I 4 \mathrm{OX}$ transgenic backgrounds. Real-time RT-qPCR analysis of total transcript levels of SR gene members of the mammalian ortholog (A) and plant-specific (B) subfamilies or of SR-like genes (C) in wild-type (Col-0), abi4-101 mutant and ABI4OX transgenic seedlings. Results are from two independent experiments and values represent means $\pm \mathrm{SE}(n=4)$. Asterisks indicate significant differences $(* p<0.05, * * p<0.01$; Student's $t$-test) from the corresponding wild type.

(A)

SR

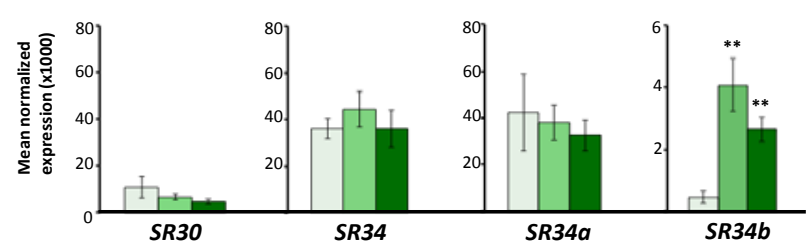

(B)

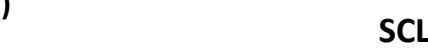

RSZ

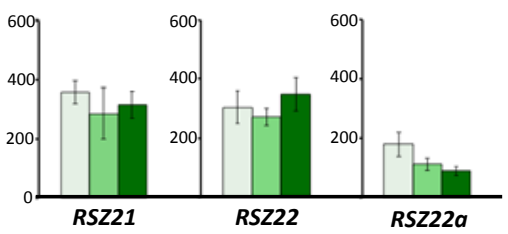

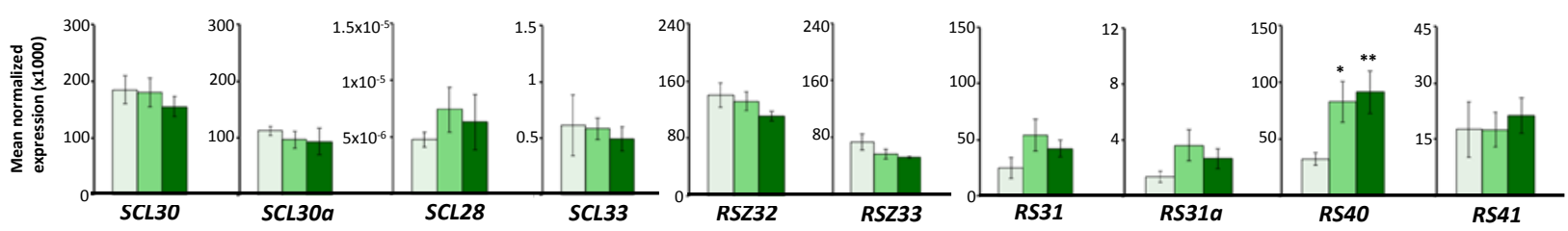

(C) SR-like
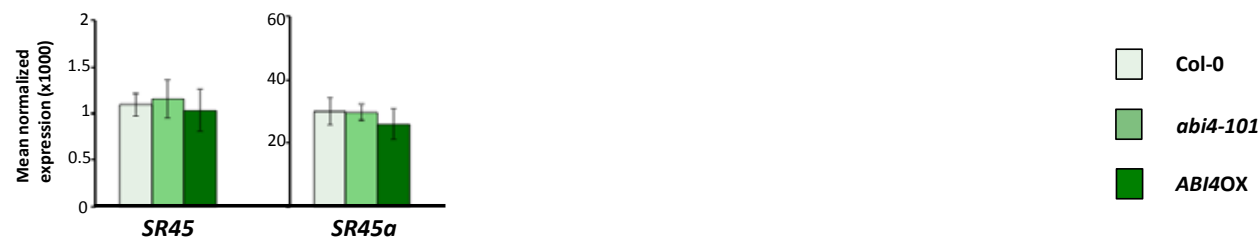

The observed induction of $S R 34 \mathrm{~b}$ and $R S 40$ in the transgenic $A B I 4 \mathrm{OX}$ background suggests a role for the ABI4 transcription factor in the activation of expression of these SR genes. However, we also observed an increase of $S R 34 b$ and $R S 40$ expression levels in the abi4-101 mutant. Given that severe mutations in the $A B I 4$ gene result in relatively weak phenotypes, it has been proposed that defects in $A B I 4$ function may be masked by genetic redundancy [77], in which case proteins with overlapping functions could compensate for a reduction in ABI4 levels and explain the sustained induction of the $S R 34 b$ and $R S 40$ genes in the abi4-101 mutant. Alternatively, upregulation of $R S 40$ and $S R 34 b$ in both the abi4-101 mutant and $A B I 4$ overexpressing lines may indicate that these SR genes are under tight ABI4 control and any fluctuations in the levels of this versatile transcription factor are sufficient to trigger their induction. In either case, our results point to the SR34b and RS40 splicing factors as promising candidates for involvement in ABI4-related functions, such as regulation of seed dormancy/germination [72], sugar signaling [78], lateral root development [79] or defense responses [80].

\subsection{ABA-Responsive cis Elements in Upstream Sequences of Arabidopsis SR and SR-Like Genes}

To better understand SR and SR-like gene expression regulation by exogenous ABA or alterations in ABA pathway components, we examined $2 \mathrm{~kb}$ of upstream sequence for each of the Arabidopsis 20 SR and SR-like genes for occurrences of previously reported ABA-responsive cis elements. 
We focused our attention on two well-characterized elements shown to confer responsiveness to ABA: the ACGT-containing ABRE and the functionally equivalent non-ACGT containing element known as Coupling Element 3 (CE3) [81-85] (Figure 6A).

Figure 6. ABA-responsive cis-element consensus sequences and their locations/orientations in the eight SR/SR-like genes responsive to ABA and/or alterations in the ABA pathway. (A) The ABRE (7 bp) and CE3 (10 bp) motif logos as generated by MEME [86] based on previously reported motifs [84]; and (B) Schematic representation of the relative position and orientation of ABRE (green) and CE3 (orange) elements found in the $2 \mathrm{~kb}$ upstream sequences of the SR or SR-like genes found to respond to any of the ABA scenarios analyzed (see Figures 2-5). The bold black arrows denote the transcription start sites. Exact coordinates of the elements are presented in Table 1.

(A) ABRE
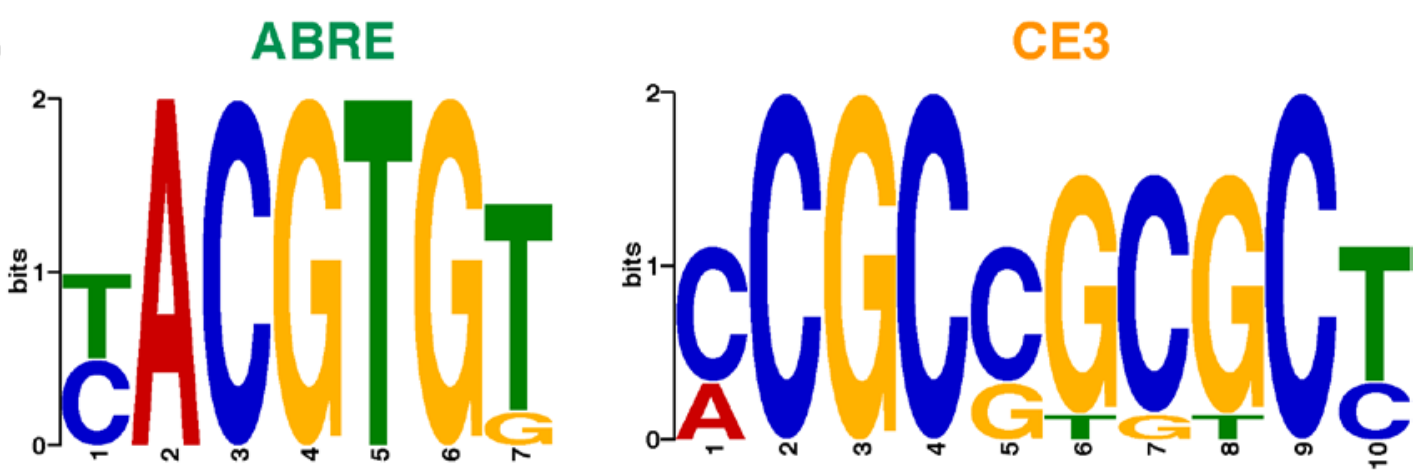

(B)

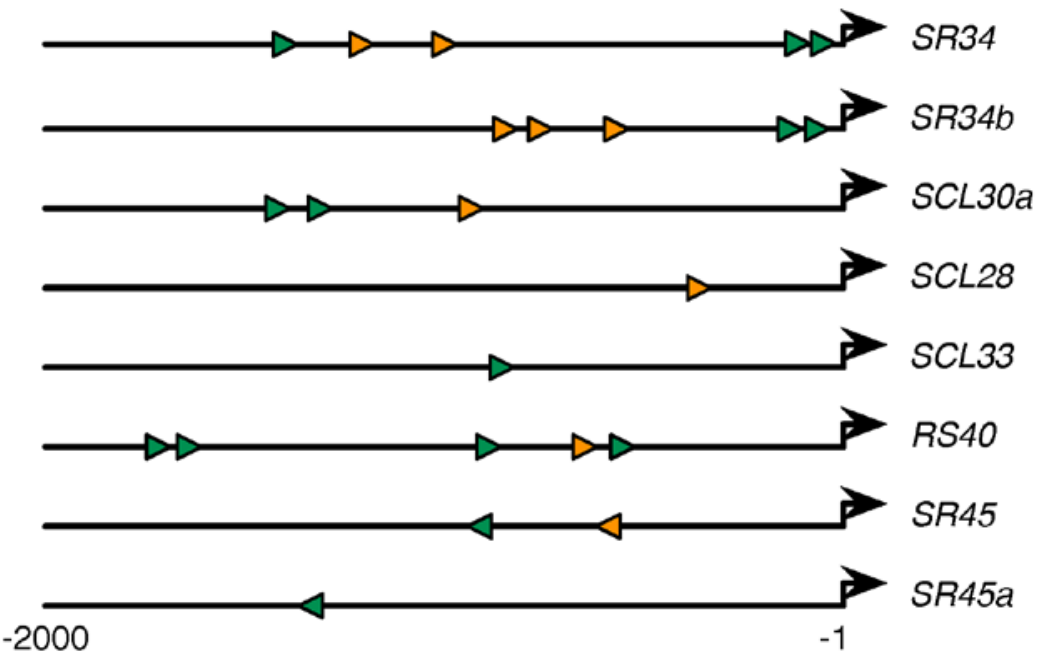

As described in the Experimental Section, we scanned the 2-kb upstream sequences of each of the 20 SR or SR-like genes for significant occurrences of ABRE and CE3 motifs. Table 1 consolidates the results of our cis-element analysis and indicates the number of each motif found, their locations and orientations in the upstream regions, and whether the gene in question responded to any of the $\mathrm{ABA}$ scenarios described above (see Figures 2-5). 
Table 1. ABA-responsive cis elements in $2 \mathrm{~kb}$ upstream sequences and corresponding expression changes under various ABA scenarios of Arabidopsis SR and SR-like genes. For each gene, the corresponding number of ABA-responsive elements (ABRE) and coupling elements (CE3) $(p<0.0001)$, their locations within $2 \mathrm{~kb}$ of upstream sequence and whether the gene responds to ABA or genetic alterations of ABA pathway components are presented. The $(+)$ and $(-)$ signs next to the gene names denote the chromosomal gene orientation. Motif locations are presented as start coordinates upstream of the translation start site; ABRE locations are shown in green, whereas CE3 locations are presented below in orange. Superscripts to the right of the motif locations indicate if the motif was found on the forward $(+)$ or reverse (-) strand of the upstream sequence. Upward- and downward-pointing arrows indicate significant induction or repression of gene expression, respectively, in response to various ABA scenarios (see Figures 2-5).

\begin{tabular}{|c|c|c|c|c|c|c|c|}
\hline Gene & \# ABRE & \# CE3 & Motif Locations (Start) & $\begin{array}{c}\text { ABA } \\
(50 \mu M)\end{array}$ & $a b a 2-1$ & abil-1 & $\begin{array}{c}\text { abi4-101 \& } \\
\text { ABI4:OX }\end{array}$ \\
\hline $\begin{array}{c}R S Z 21(-) \\
(\mathrm{AT} 1 \mathrm{G} 23860) \\
\end{array}$ & 3 & 1 & $\begin{array}{c}-593^{+},-676^{+},-1460^{-} \\
-681^{-} \\
\end{array}$ & $x$ & $x$ & $x$ & $\times$ \\
\hline $\begin{array}{c}R S Z 22(+) \\
(\mathrm{AT} 4 \mathrm{G} 31580) \\
\end{array}$ & 2 & 0 & $-166^{+},-522^{+}$ & $x$ & $x$ & $x$ & $x$ \\
\hline $\begin{array}{c}R S Z 22 a(+) \\
(\mathrm{AT} 2 \mathrm{G} 24590) \\
\end{array}$ & 1 & 0 & $-1844^{-}$ & $\times$ & $x$ & $x$ & $x$ \\
\hline $\begin{array}{c}S C 35(-) \\
(\mathrm{AT} 5 \mathrm{G} 64200) \\
\end{array}$ & 1 & 3 & $\begin{array}{c}-857^{+} \\
-745^{+},-1009^{-},-1876^{-} \\
\end{array}$ & $x$ & $x$ & $x$ & $x$ \\
\hline 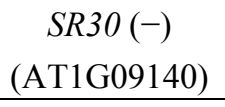 & 1 & 1 & $\begin{array}{l}-1968^{+} \\
-405^{-} \\
\end{array}$ & $x$ & $x$ & $x$ & $x$ \\
\hline $\begin{array}{c}S R 34(+) \\
(\mathrm{AT} 1 \mathrm{G} 02840) \\
\end{array}$ & 3 & 2 & $\begin{array}{l}-9^{+},-18^{+},-1394^{+} \\
-1056^{+},-1244^{+} \\
\end{array}$ & $x$ & $\uparrow$ & $x$ & $x$ \\
\hline $\begin{array}{c}S R 34 a(+) \\
(\mathrm{AT} 3 \mathrm{G} 49430)\end{array}$ & 1 & 0 & $-1276^{-}$ & $x$ & $x$ & $x$ & $x$ \\
\hline $\begin{array}{c}S R 34 b(+) \\
(\mathrm{AT} 4 \mathrm{G} 02430)\end{array}$ & 2 & 3 & $\begin{array}{c}-49^{+},-58^{+} \\
-608^{+},-780^{+},-806^{+}\end{array}$ & $x$ & $x$ & $\downarrow$ & $\uparrow$ \\
\hline $\begin{array}{c}S C L 30(+) \\
(\mathrm{AT} 3 \mathrm{G} 55460)\end{array}$ & 0 & 2 & $-1182^{+},-1481^{+}$ & $x$ & $x$ & $x$ & $x$ \\
\hline $\begin{array}{c}\text { SCL30a }(-) \\
(\mathrm{AT} 3 \mathrm{G} 13570)\end{array}$ & 2 & 1 & $\begin{array}{c}-1364^{+},-1452^{+} \\
-979^{+} \\
\end{array}$ & $\downarrow$ & $\uparrow$ & $x$ & $x$ \\
\hline $\begin{array}{c}\operatorname{SCL28(-)} \\
(\mathrm{AT5G} 18810) \\
\end{array}$ & 0 & 1 & $-353^{+}$ & $\uparrow$ & $\uparrow$ & $x$ & $x$ \\
\hline $\begin{array}{c}\text { SCL33 }(+) \\
(\mathrm{AT} 1 \mathrm{G} 55310) \\
\end{array}$ & 1 & 0 & $-834^{+}$ & $\downarrow$ & $\times$ & $x$ & $x$ \\
\hline $\begin{array}{c}R S Z 32(-) \\
(\mathrm{AT} 3 \mathrm{G} 53500) \\
\end{array}$ & 2 & 3 & $\begin{array}{c}-664^{+},-1761^{+} \\
-222^{-},-402^{-},-804^{+} \\
\end{array}$ & $x$ & $\times$ & $\times$ & $\times$ \\
\hline $\begin{array}{c}R S Z 33(-) \\
(\mathrm{AT} 2 \mathrm{G} 37340)\end{array}$ & 0 & 1 & $-1852^{+}$ & $x$ & $\times$ & $\times$ & $\times$ \\
\hline
\end{tabular}


Table 1. Cont.

\begin{tabular}{|c|c|c|c|c|c|c|c|}
\hline Gene & \# ABRE & \# CE3 & Motif Locations (Start) & $\begin{array}{c}\mathrm{ABA} \\
(\mathbf{5 0} \mu \mathrm{M})\end{array}$ & $a b a 2-1$ & $a b i 1-1$ & $\begin{array}{c}\text { abi4-101 \& } \\
\text { ABI4:OX }\end{array}$ \\
\hline $\begin{array}{c}R S 31(-) \\
(\mathrm{AT} 3 \mathrm{G} 61860)\end{array}$ & 3 & 3 & $\begin{array}{c}-188^{+},-743^{-},-1286^{-} \\
-1037^{-},-1343^{+},-1393^{+}\end{array}$ & $\times$ & $x$ & $x$ & $\times$ \\
\hline $\begin{array}{c}R S 31 a(-) \\
(\mathrm{AT} 2 \mathrm{G} 46610)\end{array}$ & 2 & 4 & $\begin{array}{c}-277^{+},-570^{-} \\
-280^{-},-614^{+},-1453^{+},-1464^{-} \\
\end{array}$ & $x$ & $\times$ & $\times$ & $x$ \\
\hline $\begin{array}{c}R S 40(+) \\
(\mathrm{AT} 4 \mathrm{G} 25500)\end{array}$ & 4 & 1 & $\begin{array}{c}-581^{+},-882^{+},-1686^{+},-1737^{+} \\
-692^{+} \\
\end{array}$ & $x$ & $\uparrow$ & $x$ & $\uparrow$ \\
\hline $\begin{array}{c}R S 41(+) \\
(\mathrm{AT} 5 \mathrm{G} 52040)\end{array}$ & 0 & 3 & $-876^{+},-1695^{+},-1892^{-}$ & $x$ & $\times$ & $x$ & $x$ \\
\hline $\begin{array}{c}S R 45(-) \\
(\mathrm{AT1G16610)}\end{array}$ & 1 & 1 & $\begin{array}{l}-939^{-} \\
-628^{-}\end{array}$ & $x$ & $\uparrow$ & $x$ & $x$ \\
\hline $\begin{array}{c}S R 45 a(-) \\
(\mathrm{AT} 1 \mathrm{G} 07350)\end{array}$ & 1 & 0 & $-1317^{-}$ & $x$ & $\uparrow$ & $\downarrow$ & $x$ \\
\hline
\end{tabular}

Of the 20 SR and SR-like genes analyzed, each gene's upstream region had at least one of the cis elements present. A total of $30 \mathrm{ABRE}$ and $30 \mathrm{CE} 3$ elements were detected, with 25/30 ABREs and $26 / 30 \mathrm{CE} 3 \mathrm{~s}$ falling within the first $1.5 \mathrm{~kb}$ of the upstream sequence. While it was previously reported that CE3 elements were sparse in the Arabidopsis genome [84], the relatively high occurrence of CE3 elements found here suggests that either the initial assessment of CE3 was underestimated or that the results presented here may be an overestimate. In either scenario, the disparity is likely due to a divergence in methodology: Here we used the program Find Individual Motif Occurrences (FIMO) [87], which calculates log-likelihood ratio scores for each motif position in a given sequence and converts these scores to $p$-values, whereas previously [84] EMBOSS profit [88] was used, which does not calculate $p$-values and relies instead upon arbitrary user-defined score cutoff values. The majority of the ABRE (22/30) and CE3 (19/30) elements were found on the forward strand of the upstream sequences.

Previous studies have suggested that a single occurrence of an ABRE is insufficient for inducing gene expression in response to $\mathrm{ABA}$ and that at least another $\mathrm{ABRE}$ or coupling element is required for $\mathrm{ABA}$ responsiveness [85,89]. Interestingly, the sole gene induced by exogenous ABA treatment was SCL28 (Table 1; see also Figure 2), which harbors only a single coupling element in its upstream region (Figure 6B; Table 1). In line with this, six late embryogenesis abundant ( $L E A$ ) genes in Prunus mume were found to be induced by ABA, but all six corresponding promoters lacked ABREs, highlighting the likelihood that there are other, yet unidentified, ABA-responsive elements important in regulating gene expression in the presence of ABA [90]. The upregulation of SR34, SCL30a, SCL28, RS40, SR45 and SR45a in the aba2-1 mutant (Table 1; see also Figure 3) is also suggestive of other cis elements and/or transcription factors mediating their ABA responsiveness. The reasoning here is that ABFs typically bind to ABREs or coupling elements to induce gene expression in response to enhanced ABA levels, and the observed upregulation of the six genes in a background with a reduced ABA content suggests their repression by the phytohormone. Similarly, the downregulation of SCL33 and SCL3Oa upon exposure to exogenous ABA (Table 1; see also Figure 2) could be mediated by as yet unknown transcriptional repressors. Up to 11 different families of transcription factors are implicated in the response to ABA-related processes [91], including WRKYs that have been shown to inhibit transcription 
from the promoter of specific ABA-responsive genes [92,93]. In addition to transcription factors, ABA-mediated gene expression can also be regulated by receptors, secondary messengers, protein kinase/phosphatase cascades and chromatin-remodeling factors [91]. In agreement with this, SR34b (two ABRE and three coupling elements) and $S R 45 a$ (one ABRE) were unresponsive to exogenous ABA treatment but were downregulated in the abil-1 mutant (Table 1; see also Figures 2 and 4), which is defective in a PP2C membrane protein phosphatase critical in ABA sensing. Lastly, $S R 34 b$ and $R S 40$ expression was altered in the ABI4-related genetic backgrounds (Table 1; see also Figure 5). Each of these SR genes has a total of five ABRE/CE3 elements in their upstream regions that could be responsible for the observed expression changes (Figure 6B and Table 1). The observed upregulation of $S R 34 b$ and $R S 40$ in both the abi4-101 mutant and ABI4 overexpressor is puzzling. It may be that the dual roles of ABI4 as both an activator and repressor of transcription [75] are at play in the ABA-mediated regulation of $S R 34 b$ and $R S 40$ gene expression. A CCAC motif has been proposed to function as the principal ABI4-binding element in genes downregulated by this transcription factor [94,95]. Indeed, $S R 34 b$ contains CCAC motifs significantly enriched in its upstream region; however, this motif was not found in the upstream region of $R S 40$ (data not shown). It is nonetheless plausible that another ABI4 binding site could confer negative regulation of $R S 40$ expression. Taken together, these findings highlight the importance of conducting linker-scanning mutagenesis to conclusively discern the contribution of particular cis elements to ABA-regulated SR and SR-like gene expression. The eight genes (Figure 6B) whose expression was affected by ABA and/or alterations in ABA components provide a suitable starting point for such analyses.

\section{Experimental Section}

\subsection{Plant Materials and Growth Conditions}

The Arabidopsis thaliana ecotypes Colombia (Col-0) or Landsberg erecta (Ler) were used as the wild type in this study. The aba2-1 and abi4-101 (Col-0 background) or abil-1 (Ler background) mutants were obtained from the Nottingham Arabidopsis Stock Centre (Nottingham, UK). The ABI4OX line (Col-0 background) [72] was kindly provided by Qi Xie (Chinese Academy of Sciences, Beijing, China). Seeds from the different genotypes were surface-sterilized for $10 \mathrm{~min}$ in $50 \%(\mathrm{v} / \mathrm{v})$ bleach and $0.07 \%(v / v)$ Triton X-100, stratified for three days at $4{ }^{\circ} \mathrm{C}$ in the dark (to break dormancy) and plated on MS medium (1× Murashige and Skoog (MS) salts (Duchefa Biochemie, Haarlem, The Netherlands), $2.5 \mathrm{mM}$ 4-morpholineethanesulfonic acid (MES) ( $\mathrm{pH} 5.7), 0.5 \mathrm{mM}$ myo-inositol and $0.8 \%$ ( $w / v)$ agar), before transfer to a growth chamber under long-day ( $16 \mathrm{~h}$ light; $80 \mu \mathrm{mol} \cdot \mathrm{m}^{-2} \cdot \mathrm{s}^{-1}$ white light) conditions at $60 \%$ relative humidity and $22{ }^{\circ} \mathrm{C}$ (light period) $/ 18^{\circ} \mathrm{C}$ (dark period). For all analyses, plant material was collected 15 days after stratification.

\subsection{ABA Treatment}

To analyze the effect of ABA treatment on SR and SR-related gene expression, 15-day old seedlings were transferred to new MS medium plates supplemented with $50 \mu \mathrm{M}$ ABA (mixed isomers, A1049; Sigma-Aldrich, St. Louis, MO, USA), with control plants being transferred to MS plates without ABA. After $5 \mathrm{~h}$ the plant material was harvested and frozen in liquid nitrogen for subsequent RNA extraction. 
The effectiveness of the ABA treatment was confirmed by RT-qPCR using $R D 29 A$ as a marker gene (see Figure 1).

\subsection{RNA Extraction and Reverse Transcription}

Total RNA was extracted from whole seedlings using the innuPREP Plant RNA kit (Analytik Jena BioSolutions, Jena, Germany) following the protocol provided. All RNA samples were digested with DNAse I (Promega, Madison, WI, USA) and phenol-chloroform purified. First strand cDNA was then synthesized using $1 \mu \mathrm{g}$ of RNA, oligo-(d)T primer and M-MLV Reverse Transcriptase (Promega) according to the manufacturer's instructions.

\subsection{Real-Time RT-qPCR Analyses}

Real-time RT-qPCR was performed using a CFX 384 Touch Real-Time PCR Detection System (Bio-Rad, Hercules, CA, USA) and the Luminaris Color HiGreen High Rox qPCR Master Mix (Thermo Scientific, Waltham, MA, USA) on $2.5 \mu \mathrm{L}$ of cDNA (diluted 1:10) per $10 \mu \mathrm{L}$ reaction volume, containing $300 \mathrm{nM}$ of each gene-specific primer (Table 2). Primers were designed to span a common region shared by all alternative transcripts annotated in TAIR and/or reported in Palusa et al. [40] and, thus, detect total expression of each SR or SR-related gene. The reaction was initiated by a Uracil-DNA Glycosylase step $\left(50{ }^{\circ} \mathrm{C}\right.$ for $\left.2 \mathrm{~min}\right)$, cycles were $95{ }^{\circ} \mathrm{C}$ for $10 \mathrm{~min}(1 \times), 95{ }^{\circ} \mathrm{C}$ for $15 \mathrm{~s} / 60{ }^{\circ} \mathrm{C}$ for $30 \mathrm{~s} / 72{ }^{\circ} \mathrm{C}$ for $30 \mathrm{~s}(40 \times)$, followed by a melting curve step to confirm the specificity of the amplified products. For each condition tested, two independent biological and technical repetitions were performed, as described by Remy et al. [96]. Data were processed using Q-Gene [97] that took the respective primer efficiency into consideration. Statistical analysis was performed using $\mathrm{R}$ software (http://www.r-project.org/), Student's $t$-test was used, and a two-sided $p$ value lower than 0.05 was accepted to indicate statistical significance.

\subsection{ABA cis-Regulatory Element Analysis}

For each of the $20 \mathrm{SR} / \mathrm{SR}$-like genes analyzed, $2 \mathrm{~kb}$ of upstream sequence was acquired from the upstream sequences dataset at TAIR (http://www.arabidopsis.org). This dataset is comprised of upstream sequences that begin either from the 5'-UTR if the gene has an annotated 5'-UTR or from the translation start site if no such annotation exists. However, in our dataset, all upstream sequences began from the transcription start site of the representative gene model for each SR or SR-like gene in TAIR. The upstream sequences were scanned using the FIMO [87] tool of the Multiple Expectation Maximization for Motif Elicitation MEME Suite [86] web server. Motif position weight matrices used for scanning were constructed using MEME [98] on the high scoring ABA-responsive element (ABRE) and coupling element (CE3) motifs previously reported [84]. Motif occurrences were considered significant if they had a $p$-value $<1 \times 10^{-4}$. If a motif occurred as a palindrome, it was counted only once. If a high scoring motif had multiple instances and these were due to a shift of a few base pairs (i.e., a subsequence of one another), only the highest scoring motif occurrence was counted. 
Table 2. Sequence of the primers used in the RT-qPCR analyses.

\begin{tabular}{|c|c|c|c|}
\hline & Gene & Primers & Sequence (5'-3') \\
\hline \multirow{2}{*}{ ABA Marker } & $R D 29 A$ & RD29AqF1 & AACGACGACAAAGGAAGTGG \\
\hline & $($ At5g52310) & RD29AqR1 & САТССТТТААТССТСССААСС \\
\hline \multicolumn{4}{|l|}{ SR Subfamilies } \\
\hline \multirow{8}{*}{ SCL } & SCL30 & SCL30qF2 & GAAGCAGATACCGATCAAGGTC \\
\hline & $(A t 3 g 55460)$ & SCL30qR2 & CTCATTGTCTCCATTTCTGTCC \\
\hline & SCL30a & SCL30aqF2 & AGATTCCAGGACAGAAGACG \\
\hline & (At3g13570) & SCL30aqR2 & TGATGTCTTTTAGCGGGAGG \\
\hline & SCL28 & SCL28qF3 & AGGCGAGAGTCAAGGCATAGTA \\
\hline & $($ At5g18810) & SCL28qR3 & GGCAAAGGAGAACGTGAAATAG \\
\hline & SCL33 & SCL33qF2 & ATCTATCTCGCCCAGGGAAG \\
\hline & $($ Atlg55310) & SCL33qR2 & GGCTCTTACCTCTGACTGGAGTT \\
\hline \multirow{4}{*}{ RS2Z } & RSZ32 & RSZ32qF1 & CCAAAGGTAGGGACCAAAGC \\
\hline & $(\operatorname{At3g} 53500)$ & RSZ32qR1 & GCAGAGTTCCTGCCATTACC \\
\hline & RSZ33 & RSZ33qF1 & GAGGAGAGATCACGCAGTCC \\
\hline & $(\mathrm{At} 2 \mathrm{G} 37340)$ & RSZ33qR1 & GCTCCCGTCTATGATCTTTGG \\
\hline \multirow{8}{*}{$\mathbf{R S}$} & $R S 31$ & RS31qF2 & CCCGAGAAGGTCTCTTAGTCC \\
\hline & $($ At3g61860) & $\mathrm{RS} 31 \mathrm{qR} 2$ & CTGTCGTATTCTGGGCTTCG \\
\hline & $R S 31 a$ & RS31aqF2 & ATGCTGGCAGTCGAAGAAGG \\
\hline & $($ At2g46610) & RS31aqR2 & AGGAGCAGGACCCTTGTACC \\
\hline & $R S 40$ & RS40qF3 & CCGTTCAAGAAGGAGAGTCC \\
\hline & $(\mathrm{At} 4 \mathrm{~g} 25500)$ & RS40qR1 & TTTCAACTTGGCCATTCTCG \\
\hline & $R S 41$ & RS41qF2 & GGTCAAGGTCGAAGTCAAGC \\
\hline & $($ At5g52040) & RS41qR2 & GCTCCATCGTATCCTCTTCC \\
\hline \multirow{6}{*}{ RSZ } & $R S Z 21$ & RSZ21qF2 & AGGCGTAGAAGCCCTAGTCC \\
\hline & (At1g23860) & RSZ21qR2 & GCGAGGTGGAGTAACACTGC \\
\hline & RSZ22 & RSZ22qF1 & CCGCAGCTACAGTAGATCACC \\
\hline & $(A t 4 g 31580)$ & RSZ22qR1 & TCTGCGTCTTTCTTTCAGTCC \\
\hline & $R S Z 22 a$ & RSZ22aqF1 & CCTCCAAGACGTCGTAGTCC \\
\hline & $($ At2g24590) & RSZ22aqR1 & CTGCGCACATCTTTCAGACC \\
\hline \multirow{2}{*}{ SC } & $S C 35$ & $\mathrm{SC} 35 \mathrm{qF} 3$ & CACAGTCGTTCCTTGAGTGC \\
\hline & $(A t 5 \mathrm{~g} 64200)$ & $\mathrm{SC} 35 \mathrm{qR} 3$ & TGGAGACCTCTCATTGCTACG \\
\hline \multirow{8}{*}{ SR } & SR30 & SR30qF2 & GACCGTAAAGGCATGTCTGG \\
\hline & (At1g09140) & SR30qR2 & TTCAGTGGCATCAAGTTTCC \\
\hline & SR34 & SR34qF1 & AAGGCAAAGTCTTCACGTAGG \\
\hline & $($ At1g02840) & SR34qR1 & AGACGGTGACCTTGACTTCG \\
\hline & $S R 34 a$ & SR34aqF2 & GCAGAAGCAGAAGCAGAAGC \\
\hline & $(\operatorname{At3g} 49430)$ & SR34aqR2 & ATCGATCTGGAAAGGGATCG \\
\hline & $S R 34 b$ & SR34bqF4 & TTGAGGATGCTCGTGATGC \\
\hline & $(\mathrm{At4g} 02430)$ & SR34bqR4 & GTTCCACCCGTAAATGATGC \\
\hline \multirow{4}{*}{ SR-like } & $S R 45$ & $\mathrm{SR} 45 \mathrm{qF} 2$ & GCGATCACCTGATTCTCCC \\
\hline & (Atlg16610) & $\mathrm{SR} 45 \mathrm{qR} 2$ & AGATCTATATCGTCTTGGAGG \\
\hline & $S R 45 a$ & SR45aqF1 & TGACCGATCATGCTCACCC \\
\hline & $($ At1g07350) & SR45aqR1 & CTGTGCCTTCTGTAGTAACG \\
\hline
\end{tabular}




\section{Conclusions}

Given that many pre-mRNA splicing factors are beginning to be implicated in ABA-mediated stress responses, we screened the 20 Arabidopsis thaliana genes encoding SR or SR-related proteins for total expression changes induced by exogenous ABA treatment or in different genetic backgrounds with alterations in key ABA pathway components.

ABA treatment of Arabidopsis seedlings notably only affected the expression of SR genes belonging to the plant-specific SCL subfamily. In fact, three of the four SCL members had their transcript levels significantly altered, indicating that they may be direct targets of ABA-responsive transcriptional activators (SCL28) or repressors (SCL3Oa and SCL33). It should be noted, however, that gene regulation upon exogenous ABA application does not necessarily imply ABA regulation in vivo. Conversely, that expression of the vast majority of SR and SR-related genes was not directly affected by ABA treatment does not exclude the possibility that they function in ABA-related responses. For example, the SR45 splicing factor has been shown to negatively regulate sugar signaling through downregulation of the ABA pathway [16]. Nevertheless, $S C L 30 a$, which we have found is involved in ABA-mediated salt and osmotic stress responses during seed germination (unpublished results), was both repressed by exogenous ABA and induced in the ABA-deficient aba2-1 mutant background, strongly suggesting its transcriptional repression by the phytohormone.

In addition to $S C L 30 a$, the expression of another five genes was altered in $a b a 2-1$, the ABA scenario in which changes in more SR and SR-like genes were observed. As this mutant background is known to display reduced endogenous ABA levels [65-68], this setting may provide a more accurate assessment of direct in vivo regulation of gene expression by the stress hormone. Apart from two members of the SCL subfamily, SCL30a and SCL28, another plant-specific SR gene, RS40, and the mammalian ortholog SR34, as well as the two Arabidopsis SR-related genes, SR45 and SR45a, exhibited altered expression in aba2-1 mutant seedlings. Interestingly, all six genes were upregulated, suggesting ABA-mediated repression of their steady-state transcript levels, although the intriguing observation that SCL28 is also markedly induced by exogenous ABA application requires further investigation.

Fewer genes were found to have their expression changed by alterations in the levels of the ABA signaling components $\mathrm{ABI} 1$ and $\mathrm{ABI} 4$. While the $S R 34 b$ mammalian ortholog was repressed and induced in the ABA-insensitive abil-1 and abi4-101 mutant backgrounds, respectively, the plant-specific RS40 and $S R 45 a$ genes were only changed (up- and downregulated, respectively) in the abi4-101 or the abil-1 mutant. Surprisingly, both genes found to be induced in the abi4-101 background, SR34b and RS40, were also upregulated in transgenic Arabidopsis plants overexpressing ABI4, possibly owing to the ability of this transcription factor to act both as an activator and repressor of gene expression [75].

Though all Arabidopsis SR and SR-like genes were found to harbor at least one ABA-responsive cis element in their upstream regions and some contain up to six elements, our results show that the mere presence alone of such elements does not translate directly into ABA-responsive gene regulation. It is likely that many as yet undiscovered cis elements play a role in governing ABA-mediated SR gene expression. Overall, this study indicates that six SR genes and the two SR-like genes from Arabidopsis thaliana are likely involved in ABA-related responses. While $S R 34 b, R S 40$ and $S R 45 a$ are most likely downstream targets of the ABI1 PP2C phosphatase and the ABI4 transcription factor, the ABA 
responsiveness of the $S R 34, S C L 30 a, S C L 28, S C L 33$ and $S R 45$ genes appears to be independent of these ABA signaling components.

It should be noted that the present study focused on total SR/SR-like gene transcript levels and hence potential effects of ABA treatment or alterations in the ABA pathway on the AS profiles were not analyzed. The Arabidopsis SR gene family is known to undergo extensive AS-Having been estimated to generate about $95 \mathrm{mRNAs}$ [40] — Which is likely to account for significant gene expression regulation. Furthermore, many of these alternative transcripts contain PTCs, pointing to an important posttranscriptional role of NMD in fine-tuning the abundance of functional SR gene transcripts present in the cell $[35,99]$. Although a previous semi-quantitative RT-PCR analysis did not detect major ABA-induced changes in the splicing pattern of Arabidopsis SR genes [40], further studies leveraging the discriminatory power of RNA-seq will facilitate not only an isoform-specific analysis of ABA regulation of SR and SR-related genes, but also allow for a full transcriptomic/differential AS snapshot of the plant's response to ABA. Moreover, it will be possible to assess the downstream effect of ABA within the context of posttranscriptional regulation of SR genes, e.g., ABA-induced NMD of SR isoforms. Last but not least, posttranslational modifications may also play a key role in ABA regulation of plant SR proteins. Indeed, aside from the well-established phosphorylation of RS domains to regulate protein-protein interactions and subcellular localization of SR proteins [100], several other modifications such as lysine acetylation, arginine methylation, ubiquitination or SUMO conjugation have been reported in animal systems to regulate SR protein degradation, stability and localization [101]. The regulation of SR gene expression is thus a decidedly complex affair involving multiple levels of control, and the mechanisms underlying ABA-regulated expression of plant SR/SR-like proteins, as well as their involvement in the ABA pathway, await further study.

\section{Acknowledgments}

We thank Xi Qie for the ABI4-overexpressing seeds. This work was funded by Fundação para a Ciência e a Tecnologia (Grant PTDC/AGR-PRO/119058/2010 as well as PostDoctoral Fellowships SFRH/BPD/80073/2011 and SFRH/BPD/81830/2011 awarded to R.F.C. and D.N.R., respectively).

\section{Author Contributions}

Tiago M. D. Cruz, Raquel F. Carvalho and Paula Duque designed the study; Tiago M. D. Cruz and Raquel F. Carvalho performed the RT-qPCR experiments; and Dale N. Richardson conducted the cis-element promoter analysis. All authors analyzed the data and wrote the article.

\section{Conflicts of Interest}

The authors declare no conflict of interest.

\section{References}

1. Lareau, L.F.; Brooks, A.N.; Soergel, D.A.; Meng, Q.; Brenner, S.E. The coupling of alternative splicing and nonsense-mediated mRNA decay. Adv. Exp. Med. Biol. 2007, 623, 190-211. 
2. Lin, S.; Fu, X.D. SR proteins and related factors in alternative splicing. Adv. Exp. Med. Biol. 2007, $623,107-122$.

3. Manley, J.L.; Krainer, A.R. A rational nomenclature for serine/arginine-rich protein splicing factors (SR proteins). Genes Dev. 2010, 24, 1073-1074.

4. Graveley, B.R.; Hertel, K.J.; Maniatis, T. SR proteins are "locators" of the RNA splicing machinery. Curr. Biol. 1999, 9, R6-R7.

5. Bourgeois, C.F.; Lejeune, F.; Stevenin, J. Broad specificity of SR (serine/arginine) proteins in the regulation of alternative splicing of pre-messenger RNA. Prog. Nucleic Acid Res. Mol. Biol. 2004, 78, 37-88.

6. Kohtz, J.D.; Jamison, S.F.; Will, C.L.; Zuo, P.; Luhrmann, R.; Garcia-Blanco, M.A.; Manley, J.L. Protein-protein interactions and 5'-splice-site recognition in mammalian mRNA precursors. Nature 1994, 368, 119-124.

7. Long, J.C.; Caceres, J.F. The SR protein family of splicing factors: Master regulators of gene expression. Biochem. J. 2009, 417, 15-27.

8. Huang, Y.; Steitz, J.A. SRprises along a messenger's journey. Mol. Cell 2005, 17, 613-615.

9. Wu, H.; Sun, S.; Tu, K.; Gao, Y.; Xie, B.; Krainer, A.R.; Zhu, J. A splicing-independent function of SF2/ASF in microRNA processing. Mol. Cell 2010, 38, 67-77.

10. Zhong, X.Y.; Wang, P.; Han, J.; Rosenfeld, M.G.; Fu, X.D. SR proteins in vertical integration of gene expression from transcription to RNA processing to translation. Mol. Cell 2009, 35, 1-10.

11. Barta, A.; Kalyna, M.; Reddy, A.S. Implementing a rational and consistent nomenclature for serine/arginine-rich protein splicing factors (SR proteins) in plants. Plant Cell 2010, 22, 2926-2929.

12. Lopato, S.; Gattoni, R.; Fabini, G.; Stevenin, J.; Barta, A. A novel family of plant splicing factors with a Zn knuckle motif: Examination of RNA binding and splicing activities. Plant Mol. Biol. 1999, 39, 761-773.

13. Ali, G.S.; Palusa, S.G.; Golovkin, M.; Prasad, J.; Manley, J.L.; Reddy, A.S. Regulation of plant developmental processes by a novel splicing factor. PLoS One 2007, 2, e471.

14. Lopato, S.; Kalyna, M.; Dorner, S.; Kobayashi, R.; Krainer, A.R.; Barta, A. atSRp30, one of two SF2/ASF-like proteins from Arabidopsis thaliana, regulates splicing of specific plant genes. Genes Dev. 1999, 13, 987-1001.

15. Kalyna, M.; Lopato, S.; Barta, A. Ectopic expression of atRSZ33 reveals its function in splicing and causes pleiotropic changes in development. Mol. Biol. Cell 2003, 14, 3565-3577.

16. Carvalho, R.F.; Carvalho, S.D.; Duque, P. The plant-specific SR45 protein negatively regulates glucose and ABA signaling during early seedling development in Arabidopsis. Plant Physiol. 2010, 154, 772-783.

17. Chen, T.; Cui, P.; Chen, H.; Ali, S.; Zhang, S.; Xiong, L. A KH-domain RNA-binding protein interacts with FIERY2/CTD phosphatase-like 1 and splicing factors and is important for pre-mRNA splicing in Arabidopsis. PLoS Genet. 2013, 9, e1003875.

18. Simpson, C.G.; Fuller, J.; Maronova, M.; Kalyna, M.; Davidson, D.; McNicol, J.; Barta, A.; Brown, J.W. Monitoring changes in alternative precursor messenger RNA splicing in multiple gene transcripts. Plant J. 2008, 53, 1035-1048. 
19. Thomas, J.; Palusa, S.G.; Prasad, K.V.; Ali, G.S.; Surabhi, G.K.; Ben-Hur, A.; Abdel-Ghany, S.E.; Reddy, A.S. Identification of an intronic splicing regulatory element involved in auto-regulation of alternative splicing of SCL33 pre-mRNA. Plant J. 2012, doi:10.1111/tpj.12004.

20. Bell, L.R.; Horabin, J.I.; Schedl, P.; Cline, T.W. Positive autoregulation of sex-lethal by alternative splicing maintains the female determined state in Drosophila. Cell 1991, 65, 229-239.

21. Ramanathan, K.; Michael, T.H.; Jiang, G.J.; Hiel, H.; Fuchs, P.A. A molecular mechanism for electrical tuning of cochlear hair cells. Science 1999, 283, 215-217.

22. Wang, E.T.; Sandberg, R.; Luo, S.; Khrebtukova, I.; Zhang, L.; Mayr, C.; Kingsmore, S.F.; Schroth, G.P.; Burge, C.B. Alternative isoform regulation in human tissue transcriptomes. Nature 2008, 456, 470-476.

23. Pan, Q.; Shai, O.; Lee, L.J.; Frey, B.J.; Blencowe, B.J. Deep surveying of alternative splicing complexity in the human transcriptome by high-throughput sequencing. Nat. Genet. 2008, 40, $1413-1415$.

24. Kornblihtt, A.R.; Schor, I.E.; Allo, M.; Dujardin, G.; Petrillo, E.; Munoz, M.J. Alternative splicing: A pivotal step between eukaryotic transcription and translation. Nat. Rev. Mol. Cell Biol. 2013, 14, 153-165.

25. Tazi, J.; Bakkour, N.; Stamm, S. Alternative splicing and disease. Biochim. Biophys. Acta 2009, 1792, 14-26.

26. Lu, T.; Lu, G.; Fan, D.; Zhu, C.; Li, W.; Zhao, Q.; Feng, Q.; Zhao, Y.; Guo, Y.; Huang, X.; et al. Function annotation of the rice transcriptome at single-nucleotide resolution by RNA-seq. Genome Res. 2010, 20, 1238-1249.

27. International Barley Genome Sequencing, Consortium. A physical, genetic and functional sequence assembly of the barley genome. Nature 2012, 491, 711-716.

28. Marquez, Y.; Brown, J.W.; Simpson, C.; Barta, A.; Kalyna, M. Transcriptome survey reveals increased complexity of the alternative splicing landscape in Arabidopsis. Genome Res. 2012, 22, $1184-1195$.

29. Staiger, D.; Brown, J.W. Alternative splicing at the intersection of biological timing, development, and stress responses. Plant Cell 2013, 25, 3640-3656.

30. Reddy, A.S.; Marquez, Y.; Kalyna, M.; Barta, A. Complexity of the alternative splicing landscape in plants. Plant Cell 2013, 25, 3657-3683.

31. Carvalho, R.F.; Feijao, C.V.; Duque, P. On the physiological significance of alternative splicing events in higher plants. Protoplasma 2013, 250, 639-650.

32. Ali, G.S.; Reddy, A.S. Regulation of alternative splicing of pre-mRNAs by stresses. Curr. Top. Microbiol. Immunol. 2008, 326, 257-275.

33. Ner-Gaon, H.; Halachmi, R.; Savaldi-Goldstein, S.; Rubin, E.; Ophir, R.; Fluhr, R. Intron retention is a major phenomenon in alternative splicing in Arabidopsis. Plant J. 2004, 39, 877-885.

34. Kazan, K. Alternative splicing and proteome diversity in plants: The tip of the iceberg has just emerged. Trends Plant Sci. 2003, 8, 468-471.

35. Filichkin, S.A.; Priest, H.D.; Givan, S.A.; Shen, R.; Bryant, D.W.; Fox, S.E.; Wong, W.K.; Mockler, T.C. Genome-wide mapping of alternative splicing in Arabidopsis thaliana. Genome Res. 2010, 20, 45-58. 
36. Ding, F.; Cui, P.; Wang, Z.; Zhang, S.; Ali, S.; Xiong, L. Genome-wide analysis of alternative splicing of pre-mRNA under salt stress in Arabidopsis. BMC Genomics 2014, 15, 431.

37. Iida, K.; Seki, M.; Sakurai, T.; Satou, M.; Akiyama, K.; Toyoda, T.; Konagaya, A.; Shinozaki, K. Genome-wide analysis of alternative pre-mRNA splicing in Arabidopsis thaliana based on full-length cDNA sequences. Nucleic Acids Res. 2004, 32, 5096-5103.

38. Duque, P. A role for SR proteins in plant stress responses. Plant Signal. Behav. 2011, 6, 49-54.

39. Reddy, A.S. Alternative splicing of pre-messenger RNAs in plants in the genomic era. Annu. Rev. Plant Biol. 2007, 58, 267-294.

40. Palusa, S.G.; Ali, G.S.; Reddy, A.S. Alternative splicing of pre-mRNAs of Arabidopsis serine/arginine-rich proteins: Regulation by hormones and stresses. Plant J. 2007, 49, 1091-1107.

41. Barta, A.; Kalyna, M.; Lorkovic, Z.J. Plant SR proteins and their functions. Curr. Top. Microbiol. Immunol. 2008, 326, 83-102.

42. Tanabe, N.; Yoshimura, K.; Kimura, A.; Yabuta, Y.; Shigeoka, S. Differential expression of alternatively spliced mRNAs of Arabidopsis SR protein homologs, atSR30 and atSR45a, in response to environmental stress. Plant Cell Physiol. 2007, 48, 1036-1049.

43. Tuteja, N. Abscisic acid and abiotic stress signaling. Plant Signal. Behav. 2007, 2, 135-138.

44. Hong, J.H.; Seah, S.W.; Xu, J. The root of ABA action in environmental stress response. Plant Cell Rep. 2013, 32, 971-983.

45. Cutler, S.R.; Rodriguez, P.L.; Finkelstein, R.R.; Abrams, S.R. Abscisic acid: Emergence of a core signaling network. Annu. Rev. Plant Biol. 2010, 61, 651-679.

46. Hubbard, K.E.; Nishimura, N.; Hitomi, K.; Getzoff, E.D.; Schroeder, J.I. Early abscisic acid signal transduction mechanisms: Newly discovered components and newly emerging questions. Genes Dev. 2010, 24, 1695-1708.

47. Lumba, S.; Toh, S.; Handfield, L.F.; Swan, M.; Liu, R.; Youn, J.Y.; Cutler, S.R.; Subramaniam, R.; Provart, N.; Moses, A.; et al. A mesoscale abscisic acid hormone interactome reveals a dynamic signaling landscape in Arabidopsis. Dev. Cell 2014, 29, 360-372.

48. Sugliani, M.; Brambilla, V.; Clerkx, E.J.; Koornneef, M.; Soppe, W.J. The conserved splicing factor SUA controls alternative splicing of the developmental regulator ABI3 in Arabidopsis. Plant Cell 2010, 22, 1936-1946.

49. Jang, Y.H.; Park, H.Y.; Lee, K.C.; Thu, M.P.; Kim, S.K.; Suh, M.C.; Kang, H.; Kim, J.K. A homolog of splicing factor SF1 is essential for development and is involved in the alternative splicing of pre-mRNA in Arabidopsis thaliana. Plant J. 2014, 78, 591-603.

50. Li, J.; Kinoshita, T.; Pandey, S.; Ng, C.K.; Gygi, S.P.; Shimazaki, K.; Assmann, S.M. Modulation of an RNA-binding protein by abscisic-acid-activated protein kinase. Nature 2002, 418, 793-797.

51. Riera, M.; Redko, Y.; Leung, J. Arabidopsis RNA-binding protein UBA2a relocalizes into nuclear speckles in response to abscisic acid. FEBS Lett. 2006, 580, 4160-4165.

52. Kim, Y.O.; Pan, S.; Jung, C.H.; Kang, H. A zinc finger-containing glycine-rich RNA-binding protein, atRZ-1a, has a negative impact on seed germination and seedling growth of Arabidopsis thaliana under salt or drought stress conditions. Plant Cell Physiol. 2007, 48, 1170-1181.

53. Yamaguchi-Shinozaki, K.; Shinozaki, K. Characterization of the expression of a desiccation-responsive rd29 gene of Arabidopsis thaliana and analysis of its promoter in transgenic plants. Mol. Gen. Genet. 1993, 236, 331-340. 
54. Richardson, D.N.; Rogers, M.F.; Labadorf, A.; Ben-Hur, A.; Guo, H.; Paterson, A.H.; Reddy, A.S. Comparative analysis of serine/arginine-rich proteins across 27 eukaryotes: Insights into sub-family classification and extent of alternative splicing. PLoS One 2011, 6, e24542.

55. Kalyna, M.; Barta, A. A plethora of plant serine/arginine-rich proteins: Redundancy or evolution of novel gene functions? Biochem. Soc. Trans. 2004, 32, 561-564.

56. Matsui, A.; Ishida, J.; Morosawa, T.; Mochizuki, Y.; Kaminuma, E.; Endo, T.A.; Okamoto, M.; Nambara, E.; Nakajima, M.; Kawashima, M.; et al. Arabidopsis transcriptome analysis under drought, cold, high-salinity and ABA treatment conditions using a tiling array. Plant Cell Physiol. 2008, 49, 1135-1149.

57. Zeller, G.; Henz, S.R.; Widmer, C.K.; Sachsenberg, T.; Ratsch, G.; Weigel, D.; Laubinger, S. Stress-induced changes in the Arabidopsis thaliana transcriptome analyzed using whole-genome tiling arrays. Plant J. 2009, 58, 1068-1082.

58. Zhang, X.N.; Mount, S.M. Two alternatively spliced isoforms of the Arabidopsis SR45 protein have distinct roles during normal plant development. Plant Physiol. 2009, 150, 1450-1458.

59. Yoshimura, K.; Mori, T.; Yokoyama, K.; Koike, Y.; Tanabe, N.; Sato, N.; Takahashi, H.; Maruta, T.; Shigeoka, S. Identification of alternative splicing events regulated by an Arabidopsis serine/arginine-like protein, atSR45a, in response to high-light stress using a tiling array. Plant Cell Physiol. 2011, 52, 1786-1805.

60. Rizhsky, L.; Liang, H.; Shuman, J.; Shulaev, V.; Davletova, S.; Mittler, R. When defense pathways collide. The response of Arabidopsis to a combination of drought and heat stress. Plant Physiol. 2004, 134, 1683-1696.

61. Yamada, K.; Fukao, Y.; Hayashi, M.; Fukazawa, M.; Suzuki, I.; Nishimura, M. Cytosolic HSP90 regulates the heat shock response that is responsible for heat acclimation in Arabidopsis thaliana. J. Biol. Chem. 2007, 282, 37794-37804.

62. Lim, C.J.; Yang, K.A.; Hong, J.K.; Choi, J.S.; Yun, D.J.; Hong, J.C.; Chung, W.S.; Lee, S.Y.; Cho, M.J.; Lim, C.O. Gene expression profiles during heat acclimation in Arabidopsis thaliana suspension-culture cells. J. Plant Res. 2006, 119, 373-383.

63. Larkindale, J.; Vierling, E. Core genome responses involved in acclimation to high temperature. Plant Physiol. 2008, 146, 748-761.

64. Gulledge, A.A.; Roberts, A.D.; Vora, H.; Patel, K.; Loraine, A.E. Mining Arabidopsis thaliana RNA-seq data with Integrated Genome Browser reveals stress-induced alternative splicing of the putative splicing regulator SR45a. Am. J. Bot. 2012, 99, 219-231.

65. Leon-Kloosterziel, K.M.; Gil, M.A.; Ruijs, G.J.; Jacobsen, S.E.; Olszewski, N.E.; Schwartz, S.H.; Zeevaart, J.A.; Koornneef, M. Isolation and characterization of abscisic acid-deficient Arabidopsis mutants at two new loci. Plant J. 1996, 10, 655-661.

66. Rook, F.; Corke, F.; Card, R.; Munz, G.; Smith, C.; Bevan, M.W. Impaired sucrose-induction mutants reveal the modulation of sugar-induced starch biosynthetic gene expression by abscisic acid signalling. Plant J. 2001, 26, 421-433.

67. Gonzalez-Guzman, M.; Apostolova, N.; Belles, J.M.; Barrero, J.M.; Piqueras, P.; Ponce, M.R.; Micol, J.L.; Serrano, R.; Rodriguez, P.L. The short-chain alcohol dehydrogenase ABA2 catalyzes the conversion of xanthoxin to abscisic aldehyde. Plant Cell 2002, 14, 1833-1846. 
68. Cheng, W.H.; Endo, A.; Zhou, L.; Penney, J.; Chen, H.C.; Arroyo, A.; Leon, P.; Nambara, E.; Asami, T.; Seo, M.; et al. A unique short-chain dehydrogenase/reductase in Arabidopsis glucose signaling and abscisic acid biosynthesis and functions. Plant Cell 2002, 14, 2723-2743.

69. Winter, D.; Vinegar, B.; Nahal, H.; Ammar, R.; Wilson, G.V.; Provart, N.J. An "Electronic Fluorescent Pictograph" browser for exploring and analyzing large-scale biological data sets. PLoS One 2007, 2, e718.

70. Koornneef, M.; Reuling, G.; Karssen, C.M. The isolation and characterization of abscisic-acid insensitive mutants of Arabidopsis thaliana. Physiol. Plant 1984, 61, 377-383.

71. Laby, R.J.; Kincaid, M.S.; Kim, D.; Gibson, S.I. The Arabidopsis sugar-insensitive mutants sis4 and sis 5 are defective in abscisic acid synthesis and response. Plant J. 2000, 23, 587-596.

72. Shu, K.; Zhang, H.; Wang, S.; Chen, M.; Wu, Y.; Tang, S.; Liu, C.; Feng, Y.; Cao, X.; Xie, Q. ABI4 regulates primary seed dormancy by regulating the biogenesis of abscisic acid and gibberellins in Arabidopsis. PLoS Genet. 2013, 9, e1003577.

73. Umezawa, T.; Nakashima, K.; Miyakawa, T.; Kuromori, T.; Tanokura, M.; Shinozaki, K.; Yamaguchi-Shinozaki, K. Molecular basis of the core regulatory network in ABA responses: Sensing, signaling and transport. Plant Cell Physiol. 2010, 51, 1821-1839.

74. Leung, J.; Bouvier-Durand, M.; Morris, P.C.; Guerrier, D.; Chefdor, F.; Giraudat, J. Arabidopsis ABA response gene ABI1: Features of a calcium-modulated protein phosphatase. Science 1994, 264, 1448-1452.

75. Wind, J.J.; Peviani, A.; Snel, B.; Hanson, J.; Smeekens, S.C. ABI4: Versatile activator and repressor. Trends Plant Sci. 2013, 18, 125-132.

76. Hoth, S.; Morgante, M.; Sanchez, J.P.; Hanafey, M.K.; Tingey, S.V.; Chua, N.H. Genome-wide gene expression profiling in Arabidopsis thaliana reveals new targets of abscisic acid and largely impaired gene regulation in the abil-1 mutant. J. Cell Sci. 2002, 115, 4891-4900.

77. Soderman, E.M.; Brocard, I.M.; Lynch, T.J.; Finkelstein, R.R. Regulation and function of the Arabidopsis ABA-insensitive4 gene in seed and abscisic acid response signaling networks. Plant Physiol. 2000, 124, 1752-1765.

78. Huijser, C.; Kortstee, A.; Pego, J.; Weisbeek, P.; Wisman, E.; Smeekens, S. The Arabidopsis SUCROSE UNCOUPLED-6 gene is identical to ABSCISIC ACID INSENSITIVE-4: Involvement of abscisic acid in sugar responses. Plant J. 2000, 23, 577-585.

79. Shkolnik-Inbar, D.; Bar-Zvi, D. ABI4 mediates abscisic acid and cytokinin inhibition of lateral root formation by reducing polar auxin transport in Arabidopsis. Plant Cell 2010, 22, 3560-3573.

80. Kerchev, P.I.; Pellny, T.K.; Vivancos, P.D.; Kiddle, G.; Hedden, P.; Driscoll, S.; Vanacker, H.; Verrier, P.; Hancock, R.D.; Foyer, C.H. The transcription factor ABI4 is required for the ascorbic acid-dependent regulation of growth and regulation of jasmonate-dependent defense signaling pathways in Arabidopsis. Plant Cell 2011, 23, 3319-3334.

81. Hobo, T.; Asada, M.; Kowyama, Y.; Hattori, T. ACGT-containing abscisic acid response element (ABRE) and coupling element 3 (CE3) are functionally equivalent. Plant J. 1999, 19, 679-689.

82. Hattori, T.; Totsuka, M.; Hobo, T.; Kagaya, Y.; Yamamoto-Toyoda, A. Experimentally determined sequence requirement of ACGT-containing abscisic acid response element. Plant Cell Physiol. 2002, 43, 136-140. 
83. Narusaka, Y.; Nakashima, K.; Shinwari, Z.K.; Sakuma, Y.; Furihata, T.; Abe, H.; Narusaka, M.; Shinozaki, K.; Yamaguchi-Shinozaki, K. Interaction between two cis-acting elements, ABRE and DRE, in ABA-dependent expression of Arabidopsis rd29A gene in response to dehydration and high-salinity stresses. Plant J. 2003, 34, 137-148.

84. Gomez-Porras, J.L.; Riano-Pachon, D.M.; Dreyer, I.; Mayer, J.E.; Mueller-Roeber, B. Genome-wide analysis of ABA-responsive elements ABRE and CE3 reveals divergent patterns in Arabidopsis and rice. BMC Genomics 2007, 8, 260.

85. Shen, Q.; Ho, T.H. Functional dissection of an abscisic acid (ABA)-inducible gene reveals two independent ABA-responsive complexes each containing a G-box and a novel cis-acting element. Plant Cell 1995, 7, 295-307.

86. Bailey, T.L.; Boden, M.; Buske, F.A.; Frith, M.; Grant, C.E.; Clementi, L.; Ren, J.; Li, W.W.; Noble, W.S. MEME SUITE: Tools for motif discovery and searching. Nucleic Acids Res. 2009, 37, W202-W208.

87. Grant, C.E.; Bailey, T.L.; Noble, W.S. FIMO: Scanning for occurrences of a given motif. Bioinformatics 2011, 27, 1017-1018.

88. Rice, P.; Longden, I.; Bleasby, A. EMBOSS: The European Molecular Biology Open Software Suite. Trends Genet. 2000, 16, 276-277.

89. Shen, Q.; Zhang, P.; Ho, T.H. Modular nature of abscisic acid (ABA) response complexes: Composite promoter units that are necessary and sufficient for ABA induction of gene expression in barley. Plant Cell 1996, 8, 1107-1119.

90. Du, D.; Zhang, Q.; Cheng, T.; Pan, H.; Yang, W.; Sun, L. Genome-wide identification and analysis of late embryogenesis abundant (LEA) genes in Prunus mume. Mol. Biol. Rep. 2013, 40, 1937-1946.

91. Fujita, Y.; Fujita, M.; Shinozaki, K.; Yamaguchi-Shinozaki, K. ABA-mediated transcriptional regulation in response to osmotic stress in plants. J. Plant Res. 2011, 124, 509-525.

92. Xie, Z.; Zhang, Z.L.; Zou, X.; Huang, J.; Ruas, P.; Thompson, D.; Shen, Q.J. Annotations and functional analyses of the rice $W R K Y$ gene superfamily reveal positive and negative regulators of abscisic acid signaling in aleurone cells. Plant Physiol. 2005, 137, 176-189.

93. Liu, Z.Q.; Yan, L.; Wu, Z.; Mei, C.; Lu, K.; Yu, Y.T.; Liang, S.; Zhang, X.F.; Wang, X.F.; Zhang, D.P. Cooperation of three WRKY-domain transcription factors WRKY18, WRKY40, and WRKY60 in repressing two ABA-responsive genes ABI4 and ABI5 in Arabidopsis. J. Exp. Bot. 2012, 63, 6371-6392.

94. Rook, F.; Hadingham, S.A.; Li, Y.; Bevan, M.W. Sugar and ABA response pathways and the control of gene expression. Plant Cell Environ. 2006, 29, 426-434.

95. Bossi, F.; Cordoba, E.; Dupre, P.; Mendoza, M.S.; Roman, C.S.; Leon, P. The Arabidopsis ABA-INSENSITIVE (ABI) 4 factor acts as a central transcription activator of the expression of its own gene, and for the induction of $A B I 5$ and SBE2.2 genes during sugar signaling. Plant J. 2009, 59, 359-374.

96. Remy, E.; Cabrito, T.R.; Batista, R.A.; Hussein, M.A.; Teixeira, M.C.; Athanasiadis, A.; Sa-Correia, I.; Duque, P. Intron retention in the 5'-UTR of the novel ZIF2 transporter enhances translation to promote zinc tolerance in Arabidopsis. PLoS Genet. 2014, 10, e1004375. 
97. Muller, P.Y.; Janovjak, H.; Miserez, A.R.; Dobbie, Z. Processing of gene expression data generated by quantitative real-time RT-PCR. Biotechniques 2002, 32, 1372-1374, 1376, 1378-1379.

98. Bailey, T.L.; Elkan, C. Fitting a mixture model by expectation maximization to discover motifs in biopolymers. Proc. Int. Conf. Intell. Syst. Mol. Biol. 1994, 2, 28-36.

99. Palusa, S.G.; Reddy, A.S. Extensive coupling of alternative splicing of pre-mRNAs of serine/arginine (SR) genes with nonsense-mediated decay. New Phytol. 2010, 185, 83-89.

100. Stamm, S. Regulation of alternative splicing by reversible protein phosphorylation. J. Biol. Chem. 2008, 283, 1223-1227.

101. Risso, G.; Pelisch, F.; Quaglino, A.; Pozzi, B.; Srebrow, A. Regulating the regulators: Serine/arginine-rich proteins under scrutiny. IUBMB Life 2012, 64, 809-816.

(C) 2014 by the authors; licensee MDPI, Basel, Switzerland. This article is an open access article distributed under the terms and conditions of the Creative Commons Attribution license (http://creativecommons.org/licenses/by/4.0/). 